PROCEEDINGS OF THE

AMERICAN MATHEMATICAL SOCIETY

Volume 132, Number 7, Pages 2115-2119

S 0002-9939(04)07395-2

Article electronically published on February 12, 2004

\title{
THE POSITIVITY \\ OF LINEAR FUNCTIONALS ON CUNTZ ALGEBRAS ASSOCIATED TO UNIT VECTORS
}

\author{
JUNG-RYE LEE AND DONG-YUN SHIN
}

(Communicated by David R. Larson)

\begin{abstract}
We study the linear functional $\rho$ on the Cuntz algebra $\mathcal{O}_{n}$ associated to a sequence $\left\langle\eta_{m}\right\rangle$ of unit vectors $\eta_{m}$ in $\mathbb{C}^{n}$ that is a generalization of the Cuntz state. We prove that $\rho$ is positive if and only if $\left\langle\eta_{m}\right\rangle$ is a constant sequence.
\end{abstract}

\section{INTRODUCTION}

For $n=2,3, \ldots$, the Cuntz algebra $\mathcal{O}_{n}$ is a simple infinite $C^{*}$-algebra generated by isometries $s_{1}, s_{2}, \ldots, s_{n}$ satisfying the Cuntz relations $s_{i}^{*} s_{j}=\delta_{i j} I$ and $\sum s_{i} s_{i}^{*}=$ $I$ (see [2]). A UHF algebra is a uniformly hyperfinite algebra and a $\mathrm{UHF}_{n}$ algebra is a UHF algebra with Glimm type $n^{\infty}$. J. Glimm [3] showed that a UHF algebra is a $C^{*}$-algebra that is the norm closure of an increasing sequence of type $I_{n_{m}}$-factors. In other words, UHF can be identified with $\bigotimes_{m=1}^{\infty} \mathcal{M}_{n_{m}}$, where $\mathcal{M}_{n_{m}}$ is an $n_{m} \times n_{m}$ matrix algebra, and $\mathrm{UHF}_{n}$ is the UHF algebra $\bigotimes_{m=1}^{\infty} \mathcal{M}_{n}$. Let $\mathcal{D}_{n}$ be the canonical diagonal subalgebra of $\mathrm{UHF}_{n}$. We consider $\mathrm{UHF}_{n}$ as a subalgebra of $\mathcal{O}_{n}$ and $\mathcal{D}_{n}$ as a maximal abelian subalgebra of $\mathcal{O}_{n}$. Since $\mathcal{O}_{n}$ is the closure of the linear span of $s_{i_{1}} s_{i_{2}} \cdots s_{i_{k}} s_{j_{l}}^{*} s_{j_{l-1}}^{*} \cdots s_{j_{1}}^{*}$, it follows that a subalgebra $\mathrm{UHF}_{n}$ is the closure of the linear span of $s_{i_{1}} s_{i_{2}} \cdots s_{i_{k}} s_{j_{k}}^{*} s_{j_{k-1}}^{*} \cdots s_{j_{1}}^{*}$ and a subalgebra $\mathcal{D}_{n}$ is the closure of the linear span of $s_{i_{1}} s_{i_{2}} \cdots s_{i_{k}} s_{i_{k}}^{*} s_{i_{k-1}}^{*} \cdots s_{i_{1}}^{*}$.

One part of the study of the Cuntz algebra is representations of $\mathcal{O}_{n}$. Representations of a $C^{*}$-algebra are related to states of it, and irreducible representations correspond to pure states by GNS constructions. As is known, states are linear functionals, and linear functionals on abelian algebras and nonabelian algebras have been studied (see [3, 4, 5]). On the other hand, product pure states of $\mathrm{UHF}_{n}$ can be described by sequences of unit vectors in $\mathbb{C}^{n}$, and extensions of these states to $\mathcal{O}_{n}$ become linear functionals on $\mathcal{O}_{n}$.

In this paper, we deal with a natural extension of product pure states of $\mathrm{UHF}_{n}$ associated to sequences of unit vectors in $\mathbb{C}^{n}$ to $\mathcal{O}_{n}$. To do this, for a sequence $\left\langle\eta_{m}\right\rangle$ of unit vectors $\eta_{m}$ in $\mathbb{C}^{n}$, we will define the associated linear functional $\rho$ on $\mathcal{O}_{n}$.

Received by the editors February 25, 2003 and, in revised form, April 17, 2003.

2000 Mathematics Subject Classification. Primary 46L30; Secondary 46L05.

Key words and phrases. Cuntz algebra, Cuntz state, associated linear functional.

The second author was supported by UOS-2002. 
Then it is natural to ask whether the linear functional $\rho$ is a state. In fact, states of $C^{*}$-algebras are positive linear functionals of norm one, and so our concern is the positivity of the associated linear functional $\rho$ on $\mathcal{O}_{n}$. We give a simple proof that if $\rho$ is positive, then $\left\langle\eta_{m}\right\rangle$ is a constant sequence.

\section{Preliminaries}

A state $\rho$ of a UHF algebra is a product state if $\rho$ satisfies $\rho(x y)=\rho(x) \rho(y)$ for $x \in I \otimes \cdots \otimes I \otimes \mathcal{M}_{n_{i}} \otimes I \otimes \cdots$ and $y \in I \otimes \cdots \otimes I \otimes \mathcal{M}_{n_{j}} \otimes I \otimes \cdots \quad(i \neq j)$. R. T. Powers [5] studied product states of UHF. We note that product pure states of a UHF algebra can be described in terms of unit vectors.

In fact, a pure state of a matrix algebra $\mathcal{M}_{n}$ is a vector state. So, for a pure state $\rho$ of $\mathcal{M}_{n}$, there exists a unit vector $\eta \in \mathbb{C}^{n}$ with $\rho(\cdot)=\langle\cdot \eta, \eta\rangle$.

Conversely, for a sequence $\left\langle\eta_{m}\right\rangle$ of unit vectors $\eta_{m} \in \mathbb{C}^{n}$, if we consider vector states $\omega_{m}$ of $\mathcal{M}_{n}$ defined by $\omega_{m}(\cdot)=\left\langle\cdot \eta_{m}, \eta_{m}\right\rangle$, then we get a product pure state $\bigotimes_{m=1}^{\infty} \omega_{m}$ of $\bigotimes_{m=1}^{\infty} \mathcal{M}_{n}$. Since an element $s_{i_{1}} \cdots s_{i_{k}} s_{j_{k}}^{*} \cdots s_{j_{1}}^{*}$ in a subalgebra $\mathrm{UHF}_{n}$ of $\mathcal{O}_{n}$ can be identified with $E_{i_{1} j_{1}} \otimes \cdots \otimes E_{i_{k} j_{k}} \otimes I \otimes \cdots$, where $E_{i j}$ is the matrix in $\mathcal{M}_{n}$ whose $(i, j)$-component is 1 and the others are 0 , we have the following:

$$
\begin{aligned}
& \left(\bigotimes_{m=1}^{\infty} \omega_{m}\right)\left(E_{i_{1} j_{1}} \otimes E_{i_{2} j_{2}} \otimes \cdots \otimes E_{i_{k} j_{k}} \otimes I \otimes \cdots\right) \\
& =\eta_{1}^{i_{1}} \overline{\eta_{1}^{j_{1}}} \eta_{2}^{i_{2}} \overline{\eta_{2}^{j_{2}}} \cdots \eta_{k}^{i_{k}} \overline{\eta_{k}^{j_{k}}}=\eta_{1}^{i_{1}} \eta_{2}^{i_{2}} \cdots \eta_{k}^{i_{k}} \overline{\eta_{k}^{j_{k}} \cdots \eta_{2}^{j_{2}} \eta_{1}^{j_{1}}} .
\end{aligned}
$$

Here, we define the associated linear functional on $\mathcal{O}_{n}$ for a given sequence of unit vectors in $\mathbb{C}^{n}$.

Definition 2.1. For a sequence $\left\langle\eta_{m}\right\rangle$ of unit vectors $\eta_{m}=\left(\eta_{m}^{1}, \eta_{m}^{2}, \ldots, \eta_{m}^{n}\right)$ in $\mathbb{C}^{n}$, the associated linear functional $\rho$ on $\mathcal{O}_{n}$ is defined by

$$
\rho\left(s_{i_{1}} s_{i_{2}} \cdots s_{i_{k}} s_{j_{l}}^{*} s_{j_{l-1}}^{*} \cdots s_{j_{1}}^{*}\right)=\eta_{1}^{i_{1}} \eta_{2}^{i_{2}} \cdots \eta_{k}^{i_{k}} \overline{\eta_{l}^{j_{l}} \eta_{l-1}^{j_{l-1}} \cdots \eta_{1}^{j_{1}}} .
$$

We now recall that for a unit vector $\eta=\left(\eta^{1}, \eta^{2}, \ldots, \eta^{n}\right) \in \mathbb{C}^{n}$, the Cuntz state $\omega_{\eta}$ is a pure state of $\mathcal{O}_{n}$ defined by

$$
\omega_{\eta}\left(s_{i_{1}} s_{i_{2}} \cdots s_{i_{k}} s_{j_{l}}^{*} s_{j_{l-1}}^{*} \cdots s_{j_{1}}^{*}\right)=\eta^{i_{1}} \eta^{i_{2}} \cdots \eta^{i_{k}} \overline{\eta^{j_{l}} \eta^{j_{l-1}} \cdots \eta^{j_{1}}},
$$

and for more details, we refer to [1]. The restriction of the Cuntz state $\omega_{\eta}$ to a subalgebra $\mathrm{UHF}_{n}$ of $\mathcal{O}_{n}$ becomes a product pure state of $\mathrm{UHF}_{n}$, and for a constant sequence $\langle\eta\rangle$ of a unit vector $\eta$ in $\mathbb{C}^{n}$, the associated linear functional is just the Cuntz state $\omega_{\eta}$.

In Theorem 3.1, we prove that if the linear functional $\rho$ associated to a sequence $\left\langle\eta_{m}\right\rangle$ of unit vectors in $\mathbb{C}^{n}$ is positive, then $\left\langle\eta_{m}\right\rangle$ is a constant sequence. Therefore we conclude that if the associated linear functional on $\mathcal{O}_{n}$ is a state, then it becomes the Cuntz state.

For the Cuntz algebra $\mathcal{O}_{n}$, an element $P \in \mathcal{O}_{n}$ of the form $s_{i_{1}} s_{i_{2}} \cdots s_{i_{k}}$ is called a monomial with length $k$, and $S_{k}$ denotes the set of all monomials in $\mathcal{O}_{n}$ with length $k$. So an element $s_{i_{1}} s_{i_{2}} \cdots s_{i_{k}} s_{j_{l}}^{*} s_{j_{l-1}}^{*} \cdots s_{j_{1}}^{*} \in \mathcal{O}_{n}$ is expressed by $P Q^{*}$ with $P=s_{i_{1}} s_{i_{2}} \cdots s_{i_{k}}$ and $Q=s_{j_{1}} s_{j_{2}} \cdots s_{j_{l}}$, and the set of all finite sums $\sum \lambda_{i} P_{i} Q_{i}^{*}$, where the $P_{i}$ 's and $Q_{i}$ 's are monomials in $\mathcal{O}_{n}$ and $\lambda_{i} \in \mathbb{C}$, is dense in $\mathcal{O}_{n}$. For a matrix $A \in \mathcal{M}_{k}$, $\operatorname{det} A$ denotes the determinant of $A$, and $A$ is said to be positive if $A=C^{*} C$ for some matrix $C$, where the adjoint matrix $C^{*}=\left(d_{i j}\right)$ of $C=\left(c_{i j}\right)$ 
is given by $d_{i j}=\overline{c_{j i}}$. Furthermore, $\mathcal{M}_{k}\left(\mathcal{O}_{n}\right)$ denotes the set of all $k \times k$ matrices whose elements are in the Cuntz algebra $\mathcal{O}_{n}$. In addition, we recall that the inner product $\langle\xi, \eta\rangle$ of two vectors $\xi=\left(\xi^{1}, \xi^{2}, \ldots, \xi^{n}\right)$ and $\eta=\left(\eta^{1}, \eta^{2}, \ldots, \eta^{n}\right)$ in $\mathbb{C}^{n}$ is given by $\sum \xi^{i} \overline{\eta^{i}}$, and we get $|\xi|^{2}=\langle\xi, \xi\rangle$.

\section{The positivity of the associated Linear functional on $\mathcal{O}_{n}$}

In this section, we deal with the linear functional $\rho$ on $\mathcal{O}_{n}$ associated to a sequence of unit vectors in $\mathbb{C}^{n}$ and find the necessary and sufficient condition for the positivity of $\rho$. Note that the linear functional $\rho$ on $\mathcal{O}_{n}$ associated to a constant sequence is the Cuntz state, and so it is positive.

In the following theorem, we prove that the positivity of the linear functional on $\mathcal{O}_{n}$ associated to a sequence $\left\langle\eta_{m}\right\rangle$ in $\mathbb{C}^{n}$ implies that $\left\langle\eta_{m}\right\rangle$ is a constant sequence. But, since $\sum s_{i} s_{i}^{*}=I$ and so

$$
\rho(I)=\rho\left(\sum s_{i} s_{i}^{*}\right)=\sum \rho\left(s_{i} s_{i}^{*}\right)=\sum \eta_{1}^{i} \overline{\eta_{1}^{i}}=\left|\eta_{1}\right|^{2}=1,
$$

the positivity of $\rho$ is equivalent to $\rho$ being a state. Consequently, we show that, for the linear functional $\rho$ on $\mathcal{O}_{n}$ associated to a sequence of unit vectors in $\mathbb{C}^{n}$, if $\rho$ is a state, then the sequence is constant.

Theorem 3.1. For a sequence $\left\langle\eta_{m}\right\rangle$ of unit vectors $\eta_{m} \in \mathbb{C}^{n}$, let $\rho$ be the associated linear functional on $\mathcal{O}_{n}$. If $\rho$ is positive, then $\left\langle\eta_{m}\right\rangle$ is a constant sequence.

To prove our theorem we need the following lemma.

For two vectors

$$
v=\left(v_{1}, v_{2}, \ldots, v_{n}\right), w=\left(w_{1}, w_{2}, \ldots, w_{n}\right) \in \mathbb{C}^{n}
$$

and $a, b, c, d \in \mathbb{C}$, let us consider a matrix $A \in \mathcal{M}_{n+2}$ given by

$$
A=\left(\begin{array}{cccccc}
a & b & v_{1} & v_{2} & \cdots & v_{n} \\
c & d & w_{1} & w_{2} & \cdots & w_{n} \\
\overline{v_{1}} & \overline{w_{1}} & 1 & 0 & \cdots & 0 \\
\overline{v_{2}} & \overline{w_{2}} & 0 & 1 & \ddots & \vdots \\
\vdots & \vdots & \vdots & \ddots & \ddots & 0 \\
\overline{v_{n}} & \overline{w_{n}} & 0 & \cdots & 0 & 1
\end{array}\right)
$$

Lemma 3.2. In the above notation, the determinant $\operatorname{det} A$ of $A$ is

$$
\left(a-|v|^{2}\right)\left(d-|w|^{2}\right)-(b-\langle v, w\rangle)(c-\langle w, v\rangle) .
$$


Proof. We have

$$
\begin{aligned}
& \operatorname{det} A=\left|\begin{array}{cccccc}
a-\overline{v_{n}} v_{n} & b-\overline{w_{n}} v_{n} & v_{1} & v_{2} & \cdots & v_{n-1} \\
c-\overline{v_{n}} w_{n} & d-\overline{w_{n}} w_{n} & w_{1} & w_{2} & \cdots & w_{n-1} \\
\overline{v_{1}} & \overline{w_{1}} & 1 & 0 & \cdots & 0 \\
\overline{v_{2}} & \overline{w_{2}} & 0 & 1 & \ddots & \vdots \\
\vdots & \vdots & \vdots & \ddots & \ddots & 0 \\
\overline{v_{n-1}} & \overline{w_{n-1}} & 0 & \cdots & 0 & 1
\end{array}\right| \\
& =\left|\begin{array}{cccccc}
a-\overline{v_{n}} v_{n}-\overline{v_{n-1}} v_{n-1} & b-\overline{w_{n}} v_{n}-\overline{w_{n-1}} v_{n-1} & v_{1} & v_{2} & \cdots & v_{n-2} \\
c-\overline{v_{n}} w_{n}-\overline{v_{n-1}} w_{n-1} & d-\overline{w_{n}} w_{n}-\overline{w_{n-1}} w_{n-1} & w_{1} & w_{2} & \cdots & w_{n-2} \\
\overline{v_{1}} & \overline{w_{1}} & 1 & 0 & \cdots & 0 \\
\overline{v_{2}} & \overline{w_{2}} & 0 & 1 & \ddots & \vdots \\
\vdots & \vdots & \vdots & \ddots & \ddots & 0 \\
\overline{v_{n-2}} & \overline{w_{n-2}} & 0 & \cdots & 0 & 1
\end{array}\right| \\
& =\cdots \\
& =\left|\begin{array}{cc}
a-|v|^{2} & b-\langle v, w\rangle \\
c-\langle w, v\rangle & d-|w|^{2}
\end{array}\right| \\
& =\left(a-|v|^{2}\right)\left(d-|w|^{2}\right)-(b-\langle v, w\rangle)(c-\langle w, v\rangle) \text {. }
\end{aligned}
$$

Now let us prove our main theorem.

Proof of Theorem 3.1. Let $\rho$ be the positive linear functional on $\mathcal{O}_{n}$ associated to a sequence $\left\langle\eta_{m}\right\rangle$ of unit vectors $\eta_{m}=\left(\eta_{m}^{1}, \eta_{m}^{2}, \ldots, \eta_{m}^{n}\right) \in \mathbb{C}^{n}$.

For $k=2,3, \ldots$, the facts $\sum_{P \in S_{k-1}} P P^{*}=I$ and $\rho(I)=1$ give

$$
\sum_{P \in S_{k-1}}|\rho(P)|^{2}=\rho\left(\sum_{P \in S_{k-1}} P P^{*}\right)=1 .
$$

Thus we can take a monomial $P \in S_{k-1}$ with $\rho(P)=\lambda(\neq 0)$. Now we consider $n+2$ monomials in $\mathcal{O}_{n}$ as follows:

$$
P_{1}=I, \quad P_{2}=P, \quad P_{3}=P s_{1}, \quad P_{4}=P s_{2}, \quad \ldots, \quad P_{n+2}=P s_{n} .
$$

The fact that $P^{*} P=I$ and the definition of $\rho$ give that the matrix $\left(\rho\left(P_{i}^{*} P_{j}\right)\right)$ in $\mathcal{M}_{k+2}$ is of the form

$$
\left(\begin{array}{cccccc}
\frac{1}{\lambda} & \lambda & \lambda \eta_{k}^{1} & \lambda \eta_{k}^{2} & \cdots & \lambda \eta_{k}^{n} \\
\frac{\lambda}{\lambda \eta_{k}^{1}} & \frac{1}{\eta_{1}^{1}} & \eta_{1}^{1} & \eta_{1}^{2} & \cdots & \eta_{1}^{n} \\
\overline{\lambda \eta_{k}^{2}} & \overline{\eta_{1}^{2}} & 0 & 1 & \ddots & \vdots \\
\vdots & \vdots & \vdots & \ddots & \ddots & 0 \\
\overline{\lambda \eta_{k}^{n}} & \frac{\eta_{1}^{n}}{0} & 0 & \cdots & 0 & 1
\end{array}\right) .
$$

On the other hand, the fact that

$$
\left(P_{i}^{*} P_{j}\right)=\left(P_{1}, \ldots, P_{k}\right)^{*}\left(P_{1}, \ldots, P_{k}\right) \in \mathcal{M}_{k}\left(\mathcal{O}_{n}\right)
$$

implies that the matrix $\left(P_{i}^{*} P_{j}\right)$ is positive. As is known, since a positive linear functional on a $C^{*}$-algebra is completely positive, the positivity of $\rho$ gives that the 
matrix $\left(\rho\left(P_{i}^{*} P_{j}\right)\right)=\rho_{k}\left(\left(P_{i}^{*} P_{j}\right)\right)$ is positive, where $\rho_{k}$ is the linear functional on $\mathcal{M}_{k}\left(\mathcal{O}_{n}\right)$ defined by $\rho_{k}\left(\left(x_{i j}\right)\right)=\left(\rho\left(x_{i j}\right)\right) \in \mathcal{M}_{k}$ for all $\left(x_{i j}\right) \in \mathcal{M}_{k}\left(\mathcal{O}_{n}\right)$.

From the positivity of $\left(\rho\left(P_{i}^{*} P_{j}\right)\right)$, we get $\operatorname{det}\left(\rho\left(P_{i}^{*} P_{j}\right)\right) \geq 0$, and by Lemma 3.2, we have the following inequality:

$$
\left(1-|\lambda|^{2}\left|\eta_{k}\right|^{2}\right)\left(1-\left|\eta_{1}\right|^{2}\right)-\left(\lambda-\left\langle\lambda \eta_{k}, \eta_{1}\right\rangle\right)\left(\bar{\lambda}-\left\langle\eta_{1}, \lambda \eta_{k}\right\rangle\right) \geq 0 .
$$

Since $\left|\eta_{1}\right|=1$, the above inequality is equal to $-\left|\lambda-\left\langle\lambda \eta_{k}, \eta_{1}\right\rangle\right|^{2} \geq 0$. Thus we obtain $|\lambda|^{2}\left|1-\left\langle\eta_{k}, \eta_{1}\right\rangle\right|^{2}=0$. Therefore $\eta_{1}$ and $\eta_{k}$ are two unit vectors with $\left\langle\eta_{k}, \eta_{1}\right\rangle=1$, which implies $\eta_{1}=\eta_{k}$.

Hence, for any $k=2,3, \ldots$, we have $\eta_{1}=\eta_{k}$, which implies that $\left\langle\eta_{m}\right\rangle$ is a constant sequence.

We have already noted that for a unit vector $\eta \in \mathbb{C}^{n}$ the Cuntz state $\omega_{\eta}$ is the linear functional on $\mathcal{O}_{n}$ associated to a constant sequence $\langle\eta\rangle$. Conversely, thanks to Theorem 3.1, we conclude that for the linear functional $\rho$ on $\mathcal{O}_{n}$ associated to a sequence of unit vectors in $\mathbb{C}^{n}$, if $\rho$ is a state, then $\rho$ must be a Cuntz state. Thus we obtain the following result.

Corollary 3.3. Let $\rho$ be an associated linear functional on $\mathcal{O}_{n}$. Then $\rho$ is a state if and only if $\rho$ is the Cuntz state.

\section{REFERENCES}

1. O. Bratteli, P. E. T. Jorgensen, and G. L. Price, Endomorphisms of $B(\mathcal{H})$, Proc. Sympos. Pure Math. 59 (1996), 93-138. MR 97h:46107

2. J. Cuntz, Simple $C^{*}$-algebras generated by isometries, Commun. Math. Phys. 57 (1977), 173185. MR 57:7189

3. J. Glimm, On a certain class of operator algebras, Trans. Amer. Math. Soc. 95 (1960), 318-340. MR 22:2915

4. S. Kakutani, On equivalence of infinite product measures, Ann. Math. 49 (1948), 214-224. MR 9:340e

5. R. T. Powers, Representations of uniformly hyperfinite algebras and their associated von Neumann rings, Ann. Math. 86 (1967), 138-171. MR 36:1989

Department of Mathematics, Daejin University, Kyeonggi, 487-711, Korea

E-mail address: jrlee@daejin.ac.kr

Department of Mathematics, University of Seoul, Seoul, 130-743, Korea

E-mail address: dyshin@uos.ac.kr 